\title{
ON THE POLAR EQUATIONS FOR LINEAR SYSTEMS AND RELATED NONLINEAR MATRIX DIFFERENTIAL EQUATIONS $\left({ }^{1}\right)$ \\ BY \\ GLEN J. CULLER (2)
}

0. Introduction. This paper is devoted to laying the groundwork for a theory of matrix differential equations and associated linear systems. The fundamental problem is that of characterizing the solutions of general linear systems from the coefficient matrices themselves. Classically this requires a study cf growth and decay properties, oscillation properties, and expansion properties; for the general problem the corresponding theories are far from complete. To develop convenient tools we present an elementary treatment of the transformation properties of matrix differential expressions in a form which directly reveals the connection to classical matrix theory. Applying this we derive polar equations for the general problem which provide a basis for separate studies of oscillation properties and growth-decay properties. There is an intrinsic connection between oscillation properties and expansion properties which will be detailed in a subsequent publication.

As notation we will use Greek letters $\eta$, $\xi$, etc., to designate constant vectors, lower case English letters $x, y, u$, etc., for vector functions, upper case for matrices (whether constant or not). The letter $D$ is reserved for the formally self-adjoint differential expression $-i(d / d t)$. When $D$ is applied to a vector or matrix function it is, of course, applied to all components. Because we wish to emphasize the isometric aspect of various transformations, we will call unitary matrices "isometries" and in the case of singularity, a transformation (or its matrix representation) which preserves length on a subspace will be termed a partial isometry.

1. Matrix differential expressions. Let $A, B, C$ be $n \times n$ matrices, $A$ and $B \in \mathscr{C}[0,1]$ and $C \in \mathscr{C}^{-1}[0,1]$. We call $P=A D C+B$ a matrix differential expression on $[0,1]$. Each $P$ defines a linear transformation $P: \mathscr{C}^{1}[0,1] \rightarrow \mathscr{C}[0,1]$ from the space of differentiable $n \times n$ matrices into the space of ccntinucus $n \times n$ matrices

$$
P(Y)=A D(C Y)+B Y
$$

Received by the editors January 16, 1963 and, in revised form, September 18, 1963.

(1) Sponsored by Office of Naval Research, U.S. Navy, Contract No. Nonr 233(24) and U. S. Army Research Office (Durham) Contract No. DA-04-495-ORD-559.

(2) On leave from the University of California, Santa Barbara. 
The Lagrange adjoint (or formal adjoint) makes correspond to each $P$ another expression $P^{+}$obtained from $P$ by a formal application of ${ }^{+}$under the rule: $(S T)^{+}=T^{+} S^{+}$where $D^{+}=D$, and $A^{+}$is the conjugate transpose of the matrix $A$. Thus

$$
P=A D C+B ; \quad P^{+}=C^{+} D A^{+}+B^{+} .
$$

From this definition we obtain a simple form of the familiar Lagrange identity extended to matrix differential expressions.

Theorem( (3) 1. If $A$ and $C \in \mathscr{C}^{1}[0,1], B \in \mathscr{C}[0,1]$ and $P=A D C+B$ then

$$
D\left(Y^{+} A S C X\right)=Y^{+} P(X)-\left(P^{+}(Y)\right)^{+} X
$$

for arbitrary $X$ and $Y \in \mathscr{C}^{1}[0,1]$

Proof. From the definition of Lagrange adjoint

$$
Y^{+} P(X)-\left(P^{+}(Y)\right)^{+} X=Y^{+}(A D(C X)+B X)-\left(C^{+} D\left(A^{+} Y\right)+B^{+} Y\right)^{+} X .
$$

Now observe that $D Z$ may occur as a differential expression or as a derivative of a matrix, these will coincide only when operating on constant matrices. To distinguish these possibilities let $(D Z) X$ note a matrix multiplier operator applied to $X$, and let $[D Z]$ denote a matrix differential expression. Then $(D Z)^{+}=-\left(D Z^{+}\right)$, while $[D Z]^{+}=\left[Z^{+} D\right]$. In (1.3) above, $\left(P^{+}(Y)\right)$ is a matrix so $\left(P^{+}(Y)\right)^{+}$is just its conjugate transpose. With this in mind,

$$
Y^{+} P(X)-\left(P^{+}(Y)\right)^{+} X=\left(Y^{+} A\right) D(C X)+D\left(Y^{+} A(C X)=D\left(Y^{+} A C X\right) .\right.
$$

The Lagrange identity for one $n$th order linear equation is included in (1.3) by considering an equivalent system of $n$ linear equations; moreover, the Lagrange identity for a $k$ th order matrix differential expression (of $n \times n$ matrices) is also included in (1.3), the matrices then being of order $k n \times k n$.

As always, integration of the Lagrange identity derives for us a Green's formula:

$$
\int_{0}^{\tau}\left[Y^{+}(P(X))-\left(P^{+}(Y)\right)^{+} X\right] d t=-\left.i Y^{+} A C X\right|_{0} ^{\tau} .
$$

DefinITION 1. Two matrix differential expressions are said to be equivalent (denoted by $\doteq$ ) if the corresponding linear transformations are identical.

THEOREM 2. Two matrix differential expressions

$$
\begin{aligned}
& P_{1}=A_{1} D C_{1}+B_{1}, \quad A_{1}, B_{1} \in \mathscr{C}[0,1], C^{1} \in \mathscr{C}^{1}[0,1], \\
& P_{2}=A_{2} D C_{2}+B_{2}, \quad A_{2}, B_{2} \in \mathscr{C}[0,1], C_{2} \in \mathscr{C}^{1}[0,1],
\end{aligned}
$$

are equivalent if and only if:

(3) The beginning stages of this development correspond to those given by Reid [19]. 


$$
A_{1} C_{1}=A_{2} C_{2} ; \quad A_{1}\left(D C_{1}\right)-A_{2}\left(D C_{2}\right)=B_{2}-B_{1} .
$$

Proof. If $P_{1} Y=P_{2} Y$ for all $Y \in \mathscr{C}^{1}[0,1]$, put $Y=I$, then $A_{1}\left(D C_{1}\right)+B_{1}$ $=A_{2}\left(D C_{2}\right)+B_{2}$. Now this means that $P_{1} Y-P_{2} Y=\left(A_{1} C_{1}\right)(D Y)-\left(A_{2} C_{2}\right) D Y$ $=\left(A_{1} C_{1}-A_{2} C_{2}\right)(D Y)$. But we can choose $Y$ so that $(D Y)$ is nonsingular, $\therefore A_{1} C_{1}=A_{2} C_{2}$.

A case of frequent occurrence is $C_{1}=I$, the identity matrix, then (1.5) may be written

$$
A_{1}=A_{2} C_{2} ; \quad A_{2} D C_{2}=B_{1}-B_{2} .
$$

The criterion for Lagrange self-adjointness, (that is $P \doteq P^{+}$) is

$$
A C=C^{+} A^{+} ; \quad A(D C)-C^{+}\left(D A^{+}\right)=B^{+}-B
$$

and for $C=I$ this reduces to

$$
A=A^{+} ; \quad D A=B-B^{+} .
$$

Matrix differential expressions can be multiplied on the right by a differentiable matrix or on the left by a continuous matrix and the result is a matrix differential expression. Multiplication on the right corresponds to an allowable change of dependent variables in the matrix equation $P(X)=0$, say $X=T Z$; whereas multiplication on the left merely forms linear combinations of the original component equations.

Definition 2. Two matrix differential expressions $P, Q$ are equivalent under a transformation if there exist nonsingular matrices $S \in \mathscr{C}[0,1], T \in \mathscr{C}^{1}[0,1]$ such that $P$ and $S Q T$ are equivalent.

Definition 3. A matrix differential expression $P=A D C+B$ is nonsingular if $A C$ is nonsingular.

THEOREM 3. Every nonsingular matrix differential expression is equivalent under a transformation to the expression D. Specifically

$$
W^{+} P T \doteq D \doteq T^{+} P^{+} W,
$$

where $P(T)=0, T(0)=C^{-1}(0)$ and $P^{+}(W)=0, W(0)=\left(A^{+}(0)\right)^{-1}$.

Proof. Let $A C$ be nonsingular on $[0,1]$, with $A, B \in \mathscr{C}[0,1], C \in \mathscr{C}^{1}[0,1]$ and $P=A D C+B$. With $T$ as defined above we have for all differentiabie $Z$

$$
P T(Z)=A D(C T Z)+B T Z=(A D(C T)+B T) Z+A C T D Z .
$$

But $A D(C T)+B T=0$, so $T^{-1} C^{-1} A^{-1} P T$ is equivalent to $D$. Now suppose $A \in \mathscr{C}^{1}[0,1]$ so that $P^{+}$is defined. Then the corresponding equivalence transformation for $P^{+}$makes $W^{-1}\left(A^{+}\right)^{-1}\left(C^{+}\right)^{-1} P^{+} W$ equivalent to $D$. Now $D=D^{+}$ so these expressions are Lagrange adjoints and equivalent. By choice of initial matrices, Green's Formula (1.4) shows that $W^{+} A C T=I$ and we have (1.9). 
Thus, starting from any nonsingular expression $P$, this construction gives an equivalence transformation to its adjoint; namely, $P^{+} \doteq\left(T^{+}\right)^{-1} W^{+} P T W^{-1}$.

Matrix differential expressions can be combined in a variety of ways $\left({ }^{4}\right)$ to build up linear matrix differential equations but only very special ones of these relate in a natural way to the problems that arise in the discussion of systems of linear equations. The most important type has the form:

$$
D Y=A Y+Y B ; \quad A, B \in \mathscr{C}[0,1] .
$$

We will need several basic properties of such equations which follow rather naturally from certain transformation properties of matrix differential expressions analogous to those well known for matrices. Let $P$ and $Q$ be matrix differential expressions and let $S, T, \psi$ be nonsingular matrices on $[0,1]$ with $S \in \mathscr{C}[0,1], T, \psi \in \mathscr{C}^{1}[0,1]$ and $\psi \psi^{+}=I$.

DEFINITION 4 (EQUIVALENCES).

(a) $P$ is similar to $Q$ under $T$ if $Q=T^{-1} P T$,

(b) $P$ is congruent to $Q$ under $T$ if $Q=T^{+} P T$,

(c) $P$ is isometric to $Q$ under $\psi$ if $Q=\psi^{+} P \psi$.

THEOREM 4. If $P=D+B, B \in \mathscr{C}[0,1]$, then

(i) $P$ is similar to $D$ under $T$ if and only if $P(T)=0, T(0)$ nonsingular.

(ii) $P$ is formally self-adjoint if and only if $P$ is isometrically equivalent to $D$, i.e., $D=\psi^{+} P \psi, \psi \psi^{+}=I$.

(iii) $T^{-1} P T$ is formally self-adjoint if and only if $T^{-1} P(T)$ is Hermitian.

Proof. Let $Z \in \mathscr{C}^{1}[0,1]$, then $T^{-1} P T(Z)=D Z+T^{-1} P(T) Z$. That is,

$$
T^{-1} P T \doteq D+T^{-1} P(T) .
$$

Observe that condition (1.8) with $A=I$ applies to (1.11) which proves (i) and shows that $T^{-1} P(T)=\left(T^{-1} P(T)\right)^{+}$is the necessary and sufficient condition for $T^{-1} P T$ to be equivalent to its adjoint. To prove (ii), use Theorem 3 with $A=C=I$, then $T=W$ since $P^{+}=P$, also $W^{+} T=I$ (see (1.9)) and thus $T^{+} T=I$. For the other direction of (ii), we know that $\psi D \psi^{*}$ is self-adjoint for all isometries $\psi$ and $P \doteq \psi D \psi^{+}$by hypothesis. Hence $P \doteq P^{+}$follows from (i).

THEOREM 5. The family of all nonsingular solutions of the equation $D Y=A Y+Y B$ is precisely the same as the set of all transformation matrices $Y$ such that

$$
P \doteq Y^{-1} Q Y ; \quad P=D+B, Q=D-A ;
$$

moreover, this family is obtained by forming all products $X Z$ where $D \doteq Z P Z^{-1}$ and $D \doteq X^{-1} Q X$.

(4) For example $\Sigma_{1}^{N} A_{i}(D Y) C_{i}=\Sigma_{1}^{M} B_{k} Y E_{k}$ 
Proof. $Y^{-1} Q Y \doteq D+Y^{-1} Q(Y)$ by (1.11) and $Y^{-1} Q Y=B$ by hypothesis. This proves (1.12). Now write $Y=X Z$ in (1.12), then $Z P Z^{-1} \doteq X^{-1} Q X$ and since $Y$ is nonsingular, we can choose any nonsingular $X \in \mathscr{C}^{1}[0,1]$ and ask for conditions on $Z$. Take $Q(X)=0$, then $P\left(Z^{-1}\right)=0$ follows and by (i) of Theorem $4, D \doteq Z P Z^{-1}, D \doteq X^{-1} P X$.

Certain equations of the form $D Y=A Y+Y B$ stand out because of the particularly simple structure of their solution matrices. We distinguish two types:

(1) Isometric equations, and (2) Hermitian equations.

Definition 5. A matrix differential equation is said to be:

(1) Isometric, if every solution which is isometric at one value $t=\tau$ is also isometric for all $t \in[0,1]$;

(2) Hermitian, if every solution which is Hermitian at one value $t=\tau$ is also Hermitian for all $t \in[0,1]$.

THEOREM 6. An equation $D Y=A Y+Y B$ is isometric if and only if there exist Hermitian matrices $H_{1}, H_{2} \in \mathscr{C}[0,1]$ and a function $f(t) \in \mathscr{C}[0,1]$ such that $A=H_{1}+f(t) I, B=H_{2}-f(t) I$. It is Hermitian if and only if there is a $C \in \mathscr{C}[0,1]$ such that $A=C+f(t) I, B=-C^{+}-f(t) I$.

Proof. In Theorem 5 we can choose $Z(0)=I, X(0)=I$; then all solutions are given by $Y=X K Z, K$ a constant matrix. Suppose $A=H_{1}+f(t) I, B=H_{2}-f(t) I$; then $A Y+Y B=H_{1} Y+Y H_{2}$ for all $Y$ so we may as well choose $D X=H_{1} X$ and $D Z^{-1}=-H_{2} Z^{-1}$, but then $X$ and $Z$ are isometries. Thus $Y$ is isometric if and only if $K$ is isometric. Now suppose $D Y=A Y+Y B$ is an isometric equation; then at each $\tau \in[0,1]$ we can prescribe $Y(\tau)=\psi$ to be an arbitrary isometry and thus at each $\tau$ we must have

$$
D Y Y^{+}+Y\left(D Y^{+}\right)=A+\psi B \psi^{+}-A^{+}-\psi B^{+} \psi^{+}=0 .
$$

Then $A(\tau)-A^{+}(\tau)+\psi\left(B^{+}(\tau)-B(\tau)\right) \psi^{+}$for all isometries $\psi$, which requires that $A(\tau)-A^{+}(\tau)=\operatorname{ir}(\tau) I$ and $B^{+}(\tau)-B(\tau)=\operatorname{ir}(\tau) I$ for some real function $r(\tau)$.

To prove the second assertion, note that for sufficiency we may choose $D X=C X, D Z^{-1}=C^{+} Z^{-1}$ and thus $D Z=-Z C^{+}$. But $Z(0)=Z(0)=I$ requires that $Z=X^{+}$and the general solution is therefore $Y=X K X^{+}$. So $Y$ can be Hermitian at $t=\tau$ only if $K$ is Hermitian; but then $Y$ is Hermitian everywhere.

Now suppose $D Y=A Y+Y B$ is a Hermitian equation, then at each $\tau \in[0,1]$ we must have $A(\tau) H+H B(\tau)=-B^{+}(\tau) H-H A^{+}(\tau)$ for all nonsingular Hermitian matrices $H$, since we can prescribe $Y(\tau)=H$. But $\left(A(\tau)+B^{+}(\tau)\right) H$ $+H\left(A^{+}(\tau)+B(\tau)\right)=0$ is only possible for arbitrary $H$ if $A(\tau)+B^{+}(\tau)=\operatorname{ir}(\tau) I$ for some real function $r(\tau)$. This leads to $A(\tau)=C(\tau)+f(\tau) I, B(\tau)=-C^{+}(\tau)-f(\tau) I$ with $\operatorname{ir}(\tau)=f(\tau)-\bar{f}(\tau)$.

Henceforth we will only consider isometric equations of the form $D Y=H_{1} Y+Y H_{2}$ and Hermitian equations of the form $D Y=C Y-Y C{ }^{+}$since 
the alteration given in Theorem 6 does not affect the solution matrices. Thus, apart from trivial exceptions, we can state:

REMARK. If $P=D+B, Q=D-A$, then $D Y=A Y+Y B$ is

(1) isometric if and only if $P=P^{+}$and $Q=Q^{+}$,

(2) Hermitian if and only if $P=Q^{+}$.

We can now summarize the elementary properties thus far obtained in the following terms.

Consider the class of all $n \times n$ matrices that are nonsingular and of class $\mathscr{C}^{1}$ on $[0,1]$. These form a group $G$ under matrix multiplication and those that are initially the identity matrix comprise a normal subgroup $\mathscr{G}_{I}$. The cosets obtained from the partitioning of $\mathscr{G}$ by $\mathscr{G}_{I}$ each consists of those matrices with the same initial value, that is, the cosets can be obtained from $\mathscr{G}_{I}$ by multiplying on the right by a constant matrix.

Now let $\mathscr{E}$ be the class of all matrix differential expressions $P=A D C+B$ where $A, C \in \mathscr{G}$ and $B \in \mathscr{C}[0,1]$. Each $P \in \mathscr{E}$ defines a linear transformation from $\mathscr{G}$ into $\mathscr{C}[0,1]$. $\mathscr{E}$ is closed under multiplication on the right and left by elements of $\mathscr{G}$ and in particular every element $P \in \mathscr{E}$ has a representation of the form $P \doteq W^{-1} D T^{-1}$ where $W, T \in \mathscr{G}$ and $P(T)=0, P^{+}(W)=0$. (Note: if $P \in \mathscr{E}$ then $P^{+} \in \mathscr{E}$.) Let $\mathscr{E}_{D}$ be the subclass of $\mathscr{E}$ consisting of those expressions equivalent to expressions of the form $D+B$. There is a 1-1 correspondence between $\mathscr{E}_{D}$ and $\mathscr{G}_{I}$ obtained by " $D+B \leftrightarrow Y$ if and only if $D Y+B Y=0$ and $Y(0)=I$." Also, for every $P, Q \in \mathscr{E}_{D}$ there exists exactly one element $Y \in \mathscr{G}_{I}$ such that $Y P \doteq Q Y$, in particular with $P=D+B$ and $Q=D-A, Y$ is the solution of $D Y=A Y+Y B, Y(0)=I$. Thus all elements of $\mathscr{E}_{D}$ are similar and the similarity transformation matrices can be chosen from $\mathscr{G}_{I}$. If $P$ and $Q$ are self-adjoint then this similarity is an isometry; if $Q=P^{+}$then this similarity is effected by a Hermitian matrix.

2. Properties of the Laplace matrix transform. The Laplace operator transform,

$$
\mathscr{L}_{F}(Y)=\int_{0}^{\infty} e^{-s F} Y e^{-s F} d s,
$$

has been studied by E. Heinz $([4], 1951)$ for bounded operators on a Hilbert space. urther generalization to Banach algebras with identity has been obtained by M. Rosenblum ([24], 1956). Here we are only concerned with the case of matrices but will be interested principally in the case that $F, Y$ are matrices of functions (not involving the integration variable in (2.1)). With $Y$ held fixed $\mathscr{L}_{F}(Y)$ is a nonlinear function of $F$ defined on the set of positive definite matrices into the space of $n \times n$ matrices. In the applications to polar decompositions the nonlinear character is our main interest.

Let $\mathscr{B}_{n}$ be the algebra of $n \times n$ matrices under the matrix norm 


$$
\|T\|=\operatorname{Sup}_{|\xi|=1}|T \xi| \text {. }
$$

If $J$ is a linear transformation of $\mathscr{B}_{n}$ into $\mathscr{B}_{n}$ we will use norm $J$ as that induced by $(2.2)$,

$$
\|J\|=\operatorname{Sup}_{Y \in \mathscr{B}_{n}} \frac{\|J(Y)\|}{\|Y\|} .
$$

Under this definition the norm of a matrix considered as a matrix multiplier (transformation equals the norm (2.2) of the matrix inducing it, so no confusion should result from the two uses of \|\| .

Lemma 1. If $F(t) \in \mathscr{C}^{k}[0,1]$ is a positive definite Hermitian matrix and $Y(t) \in \mathscr{C}^{k}[0,1]$ is arbitrary, then the matrix equation:

$$
F(t) X+X F(t)=Y(t)
$$

has a unique solution $X=X(t)$ and this solution is of class $\mathscr{C}^{k}[0,1]$. In particular,

$$
X(t)=\int_{0}^{\infty} e^{-s F(t)} Y(t) e^{-s F(t)} d s=\mathscr{L}_{F(t)}(Y(t)) ;
$$

the integral exists and converges uniformly for $t \in[0,1]$.

Except for the differentiability with respect to a parameter this lemma is a special case of the main results of Heinz and Rosenblum. The differentiability follows in a routine manner from the fact that although $D F / d t$ and $F$ may not commute, we still have $\left\|d F^{n} / d t\right\| \leqq n\|d F / d t\|\|F\|^{n}$.

Regarded as a function of $Y, \mathscr{L}_{F}(Y)$ is an invertible linear transformation of the space of $n \times n$ matrices onto itself. It commutes with the multiplier transformation defined through multiplication by $F$. From Lemma 1 we have the following formulas:

$$
\begin{aligned}
\mathscr{L}_{F}(F Y) & =F \mathscr{L}_{F}(Y), \mathscr{L}_{F}(Y F)=\mathscr{L}_{F}(Y) F, \\
\mathscr{L}_{F}(F Y+Y F) & =F \mathscr{L}_{F}(Y)+\mathscr{L}_{F}(Y) F=Y, \\
\mathscr{L}_{F}^{-1}(X) & =F X+X F .
\end{aligned}
$$

Considered as a function of $F, \mathscr{L}_{F}(Y)$ satisfies a Lipschitz condition over the class of positive definite Hermitian matrices.

Lemma 2. If $W=W^{+}>0$ and $V=V^{+}>0$, then

$$
\begin{aligned}
\left\|\mathscr{L}_{W}\right\| & =\frac{1}{2}\left\|W^{-1}\right\|,\left\|\mathscr{L}_{W}^{-1}\right\|=2\|W\| \\
\left\|\mathscr{L}_{W}-\mathscr{L}_{V}\right\| & \leqq 2\left\|\mathscr{L}_{W}\right\|\left\|\mathscr{L}_{V}\right\|\|W-V\| .
\end{aligned}
$$


Proof. The definition of $\mathscr{L}_{W}$ shows that $\mathscr{L}_{W}^{-1}(I)=2 W$ and $\mathscr{L}_{W}\left(W^{-1}\right)=\frac{1}{2} W^{-2}$, thus for (2.7) it suffices to prove, for all $Y \in \mathscr{B}_{n}$, that

$$
\frac{\|Y\|}{2\|W\|} \leqq\left\|\mathscr{L}_{W}(Y)\right\| \leqq \frac{1}{2}\left\|W^{-1}\right\| \quad\|Y\| .
$$

The left hand inequality follows directly from (2.6) since $\left\|W \mathscr{L}_{W}(Y)+\mathscr{L}_{W}(Y) W\right\|$ $\leqq 2\|W\|\left\|\mathscr{L}_{W}(Y)\right\|$. To obtain the right hand inequality, notice that fcr $W=W^{+}>0$ the largest eigenvalue of $e^{-s W}, s \geqq 0$ is $e^{-s m}$, where $m$ is the least eigenvalue of $W$ (and hence $1 / m$ is the largest eigenvalue of $W^{-1}$ ). From the definition (2.1) and a norm property, we have

$$
\left\|\mathscr{L}_{W}(Y)\right\| \leqq \int_{0}^{\infty}\left\|e^{-s W} Y E^{-s W}\right\| d s \leqq\left(\int_{0}^{\infty}\left\|e^{-s W}\right\|^{2} d s\right)\|Y\|=\frac{1}{2 m}\|Y\|
$$

which establishes (2.9). The nonlinear inequality (2.8) results directly from the formula

$$
\mathscr{L}_{W}(Y)-\mathscr{L}_{V}(Y)=\mathscr{L}_{W}\left((V-W) \mathscr{L}_{V}(Y)+\mathscr{L}_{V}(Y)(V-W)\right) .
$$

To derive this formula, let $Y \in \mathscr{B}_{n}$, then

$$
W \mathscr{L}_{W}(Y)+\mathscr{L}_{W}(Y) W=Y=V \mathscr{L}_{V}(Y)+\mathscr{L}_{V}(Y) V
$$

Now subtract $W \mathscr{L}_{V}(Y)+\mathscr{L}_{V}(Y) W$ from both sides to obtain $W\left[\mathscr{L}_{W}(Y)-\mathscr{L}_{V}(Y)\right]+\left[\mathscr{L}_{W}(Y)-\mathscr{L}_{V}(Y)\right] W=(V-W) \mathscr{L}_{V}(Y)+\mathscr{L}_{V}(Y)(V-W)$, which, by the definition of $\mathscr{L}_{W}$ is equivalent to (2.10).

THEOREM 7. If $H(t)=H^{+}(t) \in \mathscr{C}[0,1]$ and $K=K^{+}>0$, then the

$$
W_{0}(t)=K ; \quad W_{k+1}(t)=K+\int_{0}^{t} \mathscr{L}_{W_{k}(\tau)}(H(\tau)) d \tau,
$$

sequence is defined on a neighborhood $0 \leqq t \leqq t_{0}$; all $W_{k}(t)$ are positive definite Hermitian and the sequence converges uniformly to $W(t)$ on this neighborhood and the limit function matrix is the unique solution of the initial value problem

$$
\frac{d W}{d t}=\mathscr{L}_{W(t)}(H(t)) ; \quad W(0)=K .
$$

This solution can be uniquely extended throughout the interval $0 \leqq t<t_{1}$, where $t_{1}$ is the least value of $t$ such that

$$
K^{2}+\int_{0}^{t_{1}} H(\tau) d \tau
$$

is a singular matrix. The solution $W(t)$ is positive definite Hermitian on $0 \leqq t<t_{1}$. 
Proof. We first show that there is a neighborhood of the origin on which all $W_{k}(t)$ are defined and positive definite Hermitian.

Let

$$
\begin{aligned}
M_{k}(t) & =\operatorname{Sup}_{0 \leqq \tau \leqq t}\left\|W_{k}(\tau)\right\|, \\
\frac{1}{\mathscr{M}_{k}(t)} & =\operatorname{Sup}_{0 \leqq \tau \leqq t}\left\|W_{k}^{-1}(\tau)\right\|, \\
h & =\operatorname{Sup}_{0 \leqq \tau \leqq 1}\|H(\tau)\|
\end{aligned}
$$

where $t$ is, of course, restricted to those values for which the right hand sides are meaningful. From Lemma 2, (2.7) we have the estimate

$$
\left\|\int_{0}^{t} \mathscr{L}_{W_{k}(\tau)}(H(\tau)) d \tau\right\| \leqq \int_{0}^{t}\left\|\mathscr{L}_{W_{k}(\tau)}(H(\tau))\right\| d \tau \leqq \frac{t h}{2 \mathscr{M}_{k}(t)}
$$

which gives the iterated estimates

$$
\mathscr{M}_{0}-\frac{t h}{2 \mathscr{M}_{k}(t)} \leqq \mathscr{M}_{k+1}(t), \quad M_{k+1}(t) \leqq M_{0}+\frac{t h}{2 \mathscr{M}_{k}(t)},
$$

by (2.11) and the triangle inequality for norms. In particular, we see recursively that $W_{k+1}(t)$ is positive definite on some sufficiently small neighborhood $0 \leqq t<\delta$ since $K>0$. Also $\mathscr{M}_{0}(t)=\mathscr{M}_{0}=\left\|K^{-1}\right\|, M_{0}(t)=M_{0}=\|K\|$ and for all matrices $Y, Z$,

$$
\left(\mathscr{M}_{0}-\|Y\|\right)\|Z\| \leqq\|(K+Y) Z\| \leqq\left(M_{0}+\|Y\|\right)\|Z\|,
$$

which gives (2.13). Now suppose

$$
\frac{t h}{\mathscr{M}_{k}(t)} \leqq \mathscr{M}_{0} \text { for } 0 \leqq t \leqq \frac{\mathscr{M}_{0}^{2}}{2 h}=t_{0}
$$

then $\frac{1}{2} \mathscr{M}_{0} \leqq \mathscr{M}_{k+1}(t)$ by $(2.13)$ and

$$
\frac{t h}{\mathscr{M}_{k+1}(t)} \leqq \frac{\left(\frac{\mathscr{M}_{0}^{2}}{2 h}\right) h}{\left(\frac{1}{2} \mathscr{M}_{0}\right)}=\mathscr{M}_{0} .
$$

Since $W_{1}(t)$ is defined on $[0,1]$ and is Hermitian we have $\frac{3}{4} \mathscr{M}_{0} \leqq \mathscr{M}_{1}(t)$ for $0 \leqq t \leqq t_{0}$ so $W_{1}(t)>0$ on this interval. The induction above then proves that $W_{k}(t)=W_{k}^{+}(t)>0$ for $0 \leqq t \leqq t_{0}$ and all $k$. Moreover we have the following uniform bounds over this interval

$$
\left\|W_{k}(t)\right\| \leqq M_{0}+\frac{\mathscr{M}_{0}}{2}, \quad\left\|W_{k}^{-1}(t)\right\| \leqq \frac{2}{\mathscr{M}_{0}} .
$$

The convergence properties now result from the nonlinear inequality (2.8) 
of Lemma 2. This is effectively a Lipschitz condition for $\mathscr{L}_{W}(H)$ as a function of $W$. As given by Lemma 2, the inequality is only known for positive definite Hermitian $V$ and $W$, whereas the usual Lipschitz condition is assumed to hold on all sufficiently small neighborhoods of the solution. We have already shown that all $X_{k}(t)$ are in the class for which (2.8) holds, so the remainder of the proof is analogous to the classical Picard's method.

(a) Convergence. Using (2.11), (2.15) and Lemma 2 we obtain

$$
W_{k+1}(t)-W_{k}(t)=\int_{0}^{t}\left[\mathscr{L}_{W_{k}(\gamma)}(H(\tau))-\mathscr{L}_{W_{k-1}(\tau)}(H(\tau))\right] d \tau
$$

$$
\operatorname{Sup}_{0 \leqq \tau \leqq t}\left\|W_{k+1}(\tau)-W_{k}(\tau)\right\| \leqq\left(\frac{2 h t}{\mathscr{M}_{0}^{2}}\right)\left(\operatorname{Sup}_{0 \leqq \tau \leqq t}\left\|W_{k}(\tau)-W_{k-1}(\tau)\right\|\right)
$$

Let $0<\rho<1$ and consider the interval $0 \leqq t \leqq \rho t_{0}$ and denote:

$$
\|F\|=\operatorname{Sup}_{0 \leqq \tau \leqq t_{0}}\|F(\tau)\|, \quad F(\tau) \in B_{n},
$$

then (2.16) may be written

$$
\left\|W_{k+1}-W_{k}\right\| \leqq \rho\left\|W_{k}-W_{k-1}\right\| \text {. }
$$

Since $\mu<1$, the series $K+\sum_{k=0}^{\infty}\left(W_{k+1}-W_{k}\right)$ converges in norm by the ratio test (2.18) and thus by the choice of norm (2.17) the sequence of partial sums converges uniformly on $0 \leqq t \leqq \rho t_{0}$. But

$$
W_{N}(t)=K+\sum_{k=0}^{N-1}\left(W_{k+1}(t)-W_{k}(t)\right)
$$

so the iteration sequence converges to a continuous limit function $W(t)$ which satisfies

$$
W(t)=K+\int_{0}^{t} \mathscr{L}_{W(t)}(H(\tau)) d \tau, \quad 0 \leqq t \leqq \rho t_{0}
$$

and is positive definite Hermitian on this interval. Moreover, applying (2.8) to $W(t), W_{k}(t)$ and using the estimates as above, we have

$$
\operatorname{Sup}_{0 \leqq \tau \leqq \rho t_{0}}\left\|\mathscr{L}_{W(\tau)}(H(\tau))-\mathscr{L}_{W_{k}(\tau)}(H(\tau))\right\| \leqq \frac{2 h}{\mathscr{M}_{0}^{2}} \operatorname{Sup}_{0 \leqq t \leqq \rho t_{0}}\left\|W(\tau)-W_{k}(\tau)\right\|
$$

and the sequence of derivatives $d W_{k} / d t$ converges uniformly to $d W / d t$.

(b) Uniqueness. We have only defined $\mathscr{L}_{W}$ for $W=W^{+}>0$ but if we relaxed this and defined $\mathscr{L}_{W}(Y)$ by means of the basic property (2.6) then the differential equation (2.12) is still meaningful. In particular, we will prove that if $V(t) \in \mathscr{C}^{1}$ is any matrix such that 


$$
\frac{d V}{d t} V(t)+V(t) \frac{d V}{d t}=H(t), \quad V(0)=K,
$$

then $V(t) \equiv W(t)$ on $0 \leqq t \leqq \delta$ for some $\delta>0$. To see this, return to Lemma 2 and observe that equation (2.10) is valid with $\mathscr{L}_{V}(H)$ replaced by $d V / d t$ from (2.19) because $W(t)$ is positive definite. Thus

$$
\mathscr{L}_{W(t)}(H(t))-\frac{d V}{d t}=\mathscr{L}_{W_{(t)}}\left[(V-W) \frac{d V}{d t}+\frac{d V}{d t}(V-W)\right],
$$

and integrating this we have

$$
\operatorname{Sup}_{0 \leqq \tau \leqq t}\|W(t)-V(t)\| \leqq t\left[\operatorname{Sup}_{0 \leqq \tau \leqq t}\left\|\mathscr{L}_{W_{(t)}}\right\|\left\|\frac{d V}{d t}\right\|\right] \operatorname{Sup}_{0 \leqq \tau \leqq t}\|W(t)-V(t)\|
$$

but for $t$ sufficiently small, this is possible only when $W(t) \equiv V(t)$.

(c) Extension. We show here that the above construction can be used to extend the solution throughout the interval $0 \leqq t<t_{1}$, where $t_{1}$ is the least value of $t$ such that

$$
U(t)=K^{2}+\int_{0}^{t} H(\tau) d \tau
$$

is singular. The matrix $U(t) \in \mathscr{C}^{1}[0,1]$ is Hermitian and for $0 \leqq t<t_{1}$ it is positive definite. From the definition of $\mathscr{L}_{W(t)}(H(t))$ equation (2.12) shows that $(d W / d t) W+W(d W / d t)=d U / d t$ for $0 \leqq t \leqq \rho t_{0}$. So $W(t)$ is a positive definite square root of $U(t)$. Now the convergence proof worked for any $0 \leqq \rho<1$ so we have $W(t)$ on $0 \leqq t<t_{0}$. If $t_{0}<t_{1}$, then let $\mathscr{M}_{1}^{2}$ be the least eigenvalue of $W(t)$ for any $0 \leqq t \leqq t_{1}$. If we now begin anew at $t=t_{0}-\mathscr{M}_{1}^{2} / 4 h$ with the initial value $K$ replaced by $W\left(t_{0}-\mathscr{M}_{1}^{2} / 4 h\right)$, then we can construct the solution on $t_{0}-\mathscr{M}_{1}^{2} / 4 h \leqq t<t_{0}+\mathscr{M}_{1}^{2} / 4 h$ by (2.14). Thus extension is possible up to $t_{1}$.

Finally, uniqueness holds on $0 \leqq t<t_{1}$ since it holds on an initial closed interval of positive width, so there can be no last point at which two solutions coincide short of $t_{1}$.

THEOREM 8. If $H(t) \in \mathscr{C}[0,1], H(t)=H^{+}(t)$, then the differential equation

$$
\frac{d W}{d t}=\mathscr{L}_{W(t)}\left(W^{2}(t) H(t)+H(t) W^{2}(t)\right), \quad W(0)=W^{+}(0)>0
$$

has a unique solution on the whole interval $[0,1]$, constructable by the Picard method of Theorem 7. The solution is positive definite and Hermitian on $[0,1]$.

Proof. By the definition of $\mathscr{L}_{W}$, we may write (2.20) as

$$
\frac{d W}{d t}=W^{2} \mathscr{L}_{W}(H)+\mathscr{L}_{W}(H) W^{2}=\mathscr{L}_{W^{2}}^{-1}\left(\mathscr{L}_{W}(H)\right),
$$


where $W=W^{+}>0$. Since $W$ and $W^{2}$ are positive definite Hermitian matrices, both $\mathscr{L}_{W}$ and $\mathscr{L}_{W^{2}}^{-1}$ are bounded transformations by Lemma 2 and satisfy restricted Lipschitz conditions in terms of $W$ and $W^{2}$. But for $H=H^{+}$, we have $d W / d t=(d W / d t)^{+}$and we can repeat all of the proof of Theorem 8 without alteration. Actually, the complication introduced by $\mathscr{L}_{W^{2}}^{-1}$ is a benefit to convergence because $\left\|\mathscr{L}_{W^{2}}^{-1}\right\|=2\|W\|^{2}$, whereas $\left\|\mathscr{L}_{W}\right\|=\frac{1}{2}\left\|W^{-1}\right\|$.

In the next section we will prove that the solution extends to the whole interval $[0,1]$ but for this we need a further property of Hermitian equations defined in $\S 1$.

3. Polar factors. In this section we will give a constructive method of determining the polar factors of a solution matrix for the general problem

$$
D Y=A Y, \quad Y(0)=I ; \quad A \in \mathscr{C}[0,1] .
$$

We should naturally look among the isometric equations to determine the isometric factors and among the Hermitian equations for the positive definite Hermitian factors. This is feasible since the Hermitian equations have a definiteness property.

REMARK 1. Every solution of a Hermitian equation $D X=B X+X C$ which is positive definite and Hermitian at one value $t=\tau$ is positive definite and Hermitian on the whole interval $[0,1]$, where $B, C \in \mathscr{C}[0,1]$.

Proof. In $\S 1$ we saw that for Hermitian equations there is a continuous matrix $A$ such that $B X+X C=A X-X A^{+}$for all $X$ and found that all solutions $X=X(t)$ are of the form

$$
X(t)=Y(t) K Y^{+}(t), \quad K \text { a constant matrix, }
$$

where $D Y=A Y$ and $Y(0)$ is nonsingular. Now if $X(\tau)=X^{+}(\tau)>0$, then $K=K^{+}>0$ since $K=Y^{-1}(\tau) x(\tau)\left(Y^{-1}(\tau)\right)^{+}$. But then $Y(t) K Y^{+}(t)>0$ for all $t \in[0,1]$ and hence $X(t)=X^{+}(t)>0$.

We may even restrict our attention to the simpler forms of isometric and Hermitian equations.

REMARK 2. All differentiable isometries $O$ are obtained as solutions of isometric equations of the form

$$
D O=H_{0} \Phi, \quad \Phi(0) \Phi^{+}(0)=I ; \quad H_{0}=H_{0}^{+} \in \mathscr{C}[0,1]
$$

and all differentiable positive definite Hermitian matrices are obtained as solutions of Hermitian equations of the form

$$
\frac{d W}{d t}=H_{1} W+W H_{1}, \quad W(0)=W^{+}(0)>0 ; \quad H_{1}=H_{1}^{+} \in \mathscr{C}[0,1] .
$$

Proof. If $\Phi \Phi^{+}=I$ and $\Phi \in \mathscr{C}^{1}[0,1]$, then $(D \Phi) \Phi^{+}$is Hermitian and continuous. If $W=W^{+}>0$ and $W \in \mathscr{C}^{1}[0,1]$ then the equation $d W / d t=X W+W X$ has a unique solution 


$$
X=\int_{0}^{\infty} e^{-s W(t)} \frac{d W}{d t} e^{-s W(t)} d s
$$

by Lemma 1 . This solution is both continuous and Hermitian since $W(t)$ and $d W / d t$ are.

The properties needed for the proposed polar decomposition arise as direct corollaries of Theorems 7 and 8. Also, we have nowhere assumed either existence or uniqueness of square roots of non-negative definite matrices and both follow from a simple limit argument and Theorem 7. Admittedly, this may only be a curiosity, but since Picard's method works it is reasonable to consider those things constructable from (2.12); particularly since the square root of a matrix still poses a challenging computational problem.

CoROllaRY 1. If $A \in \mathscr{C}^{k}[0,1]$ then the polar decomposition of every matrix $Y$ satisfying $D Y=A Y, Y(0)$ invertible, has all its polar factors of class $\mathscr{C}^{k+1}[0,1]$.

Proof. Let $Y=W \Phi=\Phi V, W=W^{+}>0, \Phi \Phi^{+}=I$ be its polar decomposition. Solve the equation

$$
\frac{d W}{d t}=\mathscr{L}_{W(t)}\left(\frac{d}{d t} Y(t) Y^{+}(t)\right), \quad W(0)=\left(Y(0) Y^{+}(0)\right)^{1 / 2} .
$$

The solution exists on the whole interval since $W^{2}(t)=Y(t) Y^{+}(t)$ is nonsingular on $[0,1]$, thus $W(t) \in \mathscr{C}^{1}[0,1]$. Now in (2.4) take the right hand side to be $(d / d t)\left(W^{2}(t)\right)$, then $X=d W / d t$ is the solution obtained in Lemma 1 with $F(t)$ replaced by $W(t)$ and is of class $\mathscr{C}^{1}[0,1]$ since $W(t)$ and $Y(t)$ are. Thus $W(t) \in \mathscr{C}^{2}[0,1]$ and so forth until $d W / d t$ is limited by the differentiability of $(d / d t)\left(W^{2}(t)\right)$. Since $W^{2}(t)$ is of class $\mathscr{C}^{k+1}[0,1]$ this process may be continued to show that $W(t)$ is of class $\mathscr{C}^{k+1}[0,1]$. Finally,

$$
\Phi=W^{-1} Y \text {, so } \Phi \in \mathscr{C}^{k+1}[0,1] \text { and similarly, } V \in \mathscr{C}^{k+1}[0,1] \text {. }
$$

In general a positive definite Hermitian matrix of class $\mathscr{C}^{k}[0,1]$ has a square root of the same differentiability by Remark 2 and the above. The positive definiteness is clearly necessary on the closed interval as can be seen by the simple counterexample in one dimension, $W(t)=t^{2 / 3}$. The differentiability properties of the square root of positive definite Hermitian matrices and related regularity questions has been carefully studied by Reid ([22], 1958) using the binomial series approach. Our method presented here is constructively different and by virtue of the exponential functions involved in constructing a solution to (2.12) may have the advantage of more rapid convergence when used as the basis for a numerical method. Although differentiability may not hold for square roots of non-negative Hermitian matrices, they can still be be constructed using the differential equation approach via (2.12). 
REMARK 3. The square root of a non-negative Hermitian matrix $Q(s)$ may be constructed by solving

$$
\frac{d W}{d t}=4 t^{3} \mathscr{L}_{W}(I-Q(s)), \quad W(-1)=I
$$

on the interval $-1 \leqq t \leqq 0$. The $\lim _{t \rightarrow 0} W(t, s)$ exists and equals $Q^{1 / 2}(s)$.

Proof. Let $U(t)=t^{4} I+\left(1-t^{4}\right) Q$ and consider the equation (3.7). For $t \in[-1,0], U(t)>0$ and $W(t)=(Y(t))^{1 / 2}$. But $t^{4}$ is a lower bound on the eigenvalue of $U(t)$ and hence $1 / t^{2}$ is an upper bound for $\left\|W^{-1}(t)\right\|$. Thus by Lemma 2 ,

$$
\left|\frac{d W}{d t}\right| \leqq 4|t|^{3}\left\|\mathscr{L}_{W}\right\|\|I-Q\|=4|t|\|I-Q\|
$$

and hence the limit from the left exists and equals $Q^{1 / 2}$.

We are now in a position to combine the foregoing properties to separate the polar factors in the solution $Y$ of (3.1). Consider first the case of constant coefficient matrix $A$ in (3.1). Write $A=H_{0}-i H_{1} ; H_{0}, H_{1}$ Hermitian. Then the solution is

$$
Y(t)=e^{t\left(H_{1}+i H_{0}\right)}
$$

and if $H_{0}, H_{1}$ commute the polar factors are

$$
W=e^{t H_{1}}, \quad \phi=e^{i t H_{0}} .
$$

In the general case, the exponential solution above need not hold and we are led to the use of the Laplace matrix transform to obtain the desired separation of polar factors. As above, write $A=H_{0}-i H_{1}$ and let $Y=\psi Z$. Then

$$
D Y=(D \psi) Z+\psi D Z=\left(H_{0}-i H_{1}\right) \psi Z
$$

and the equations for $Z, \psi$ can be written

$$
D \psi=H_{0} \psi ; \quad \frac{d Z}{d t}=H_{2} Z ; \quad H_{2}=\psi+H_{1} \psi .
$$

Choose $\psi(0)=I$, then $\psi$ is isometric since $H_{0}=H_{0}^{+}$and the problem is reduced to the decomposition of $Z$. Let the polar factors of $Z$ be $W$ and $\theta$ (i.e., $Z=W \theta$ ). Then by the definition of $\mathscr{L}_{W}$,

$$
\frac{d W}{d t}=\mathscr{L}_{W}\left(H_{2}\right) W^{2}+W^{2} \mathscr{L}_{W}\left(H_{2}\right) ; \quad W(0)=I,
$$

and $W$ can be constructed by Theorem 8 . To obtain an equation for $\theta$, observe that

$$
\frac{d \theta}{d t}=W^{-1}\left(H_{2} W-\frac{d W}{d t}\right) \theta ; \theta(0)=I
$$


Using the identity $H_{2}=\mathscr{L}_{W}\left(H_{2}\right) W+W \mathscr{L}_{W}\left(H_{2}\right)$ and (3.8) we have

$$
D \theta=i\left(W \mathscr{L}_{W}\left(H_{2}\right)-\mathscr{L}_{W}\left(H_{2}\right) W\right) \theta .
$$

In the construction of the solution of (3.8) by the Picard iteration given above, it is easy to obtain the matrix $2 W \mathscr{L}_{W}\left(\mathrm{H}_{2}\right)$. This matrix bridges the connection between the $\mathrm{Z}$ equation and its isometric factor equation.

Theorem 9. Let $H=H^{+} \in \mathscr{C}[0,1]$ and $M=2 W \mathscr{L}_{W}(H)$, where

$$
\frac{d W}{d t}=\mathscr{L}_{W}(H) W^{2}+W^{2} \mathscr{L}_{W}(H) ; \quad W(0)=I ;
$$

then the solution $\mathrm{Z}$ of

$$
\frac{d Z}{d t}=H Z ; \quad Z(0)=I
$$

has a polar decomposition $\mathrm{Z}=W \theta$, where

$$
D \theta=K \theta ; \quad \theta(0)=I
$$

and $M=H-i K$.

The completion of the decomposition of $Y$ is given by

$$
Y=\left(\psi^{+} W \psi\right)\left(\psi^{+} \theta\right) .
$$

The growth properties of the solutions are determined by $W$, which indicates the value of a detailed study of equation (3.8) and the matrix $M$ in their dependence upon $H$. The development presented here does not require the restriction to a finite interval and thus an asymptotic analysis is also suggested. Finally, it appears that this formalism may have significant advantages in numerical calculations because it so frequently happens that the rapid oscillations of component functions in $Y$ are consequences of the isometric factor in the solutions while both $M$ and $W$ are well behaved. Thus the extent to which $M$ may be used to answer questions concerning oscillation characteristics is of importance.

The author is extremely grateful to Professors M. R. Hestenes and W. T. Reid for making their works available and for their encouragement.

\section{BIBLIOGRAPHY}

1. G.D. Birkhoff, On the asymptotic character of the solutions of certain linear differential equations containing a parameter, Trans. Amer. Math. Soc. 9 (1908), 219-232.

2. - On matrices of analytic functions, Math. Ann. 74 (1913), 122.

3. E. Coddington and N. Levinson, Theory of ordinary differential equations, McGraw-Hill, New York, 1955.

4. E. Heinz, Beiträge zur Störungstheorie der Spectralzerlegung, Math. Ann. 123 (1951) 415-438.

5. M. R. Hestenes, Applications of the theory of quadratic forms in Hilbert space to the calculus of variations, Pacific J. Math. 1 (1951), 525-581. 
6. M. R. Hestenes and G. D. Birkhoff, Natural isoperimetric conditions in the calculus of variations, Duke Math. J. 1 (1935), 198.

7. M. R. Hestenes, Inversion of matrices by biorthogonalization and related results, J. Soc. Indust. Appl. Math. 6 (1958), 51-90.

8. - On a representation of general operators. (Unpublished).

9. - Sufficient conditions for the problem of Bolza in the calculus of variations, Trans. Amer. Math. Soc. 36 (1934), 793-818.

10. J. von Neumann, Allgemeine Eigenwerttheorie Hermitescher Functionaloperatoren, Math. Ann. 102 (1929), 49.

11. —— Über adjungierte Funktionaloperatoren, Ann. of Math. (2) 33 (1932), 294-310.

12. - - Some matrix-inequalities and metrization of matric-space, Forsch.-Inst. Math. Mech. Univ. Tomsk 1 (1937), 286-299.

13. A. Ostrowski, Bounds for the greatest latent root of a positive matrix, J. London Math. Soc. 27 (1952), 253-256.

14. A. Ostrowski and $O$. Taussky, On the variation of the determinant of a positive definite matrix, Nederl. Akad. Wetensch. Proc. Ser. A 54 = Indag. Math. 13 (1951), 383-385.

15. A. Ostrowski, On the spectrum of a one-parametric family of matrices, J. Reine Ange w. Math. 193 (1954), 143-160.

16. - - Uber Normen von Matrizen, Math. Z. 63 (1955), 2-18.

17. W. T. Reid, Oscillation criteria for linear differential systems with complex coefficients, Pacific J. Math. 6 (1956), 733-751.

18. - A comparison theorem for self-adjoint differential equations of second order, Ann. of Math. (2) 65 (1957), 197-202.

19. - Adjoint linear differential operators, Trans. Amer. Math. Soc. 85 (1957), 446-461.

20. - - Remarks on a matrix transformation for linear differential equations, Proc. Amer. Math. Soc. 8 (1957), 708-712.

21. - Principal solutions of non-oscillatory self-adjoint linear differential systems, Pacific J. Math. 8 (1958), 147-169.

22. - - A Prüfer transformation for differential systems, Pacific J. Math. 8 (1958), 575-584.

23. F. Riesz and B. Sz.-Nagy, Functional analysis, Ungar, New York, 1955.

24, M. Rosenblum, On the operator equation $B X-X A=Q$, Duke Math. J. 23 (1956), 263-269.

25. A. E. Taylor, Conjugations of complex linear spaces, Univ. California Publ. Math. (N. S.) 2 (No. 1, Seminar Rep. in Math. Los Angeles), (1944), 85-102.

26. - Analytic functions in general analysis, Ann. Scuola Norm. Sup. Pisa (2) 6 (1937) $277-292$.

UNIVERSITY OF CALIFoRNIA, Santa Barbara, California(5)

(5) Author on leave, at Thompson Ramo Wooldridge Inc., RW Division Research Laboratory. 